\title{
ON THE PARABOLIC GENERATOR OF A GENERAL ONE-DIMENSIONAL LÉVY PROCESS
}

NATHALIE EISENBAUM

Laboratoire de Probabilités, UMR 7599, CNRS - Université Paris VI. 4, Place Jussieu, 75252, Paris Cedex 05, France.

email: nathalie.eisenbaum@upmc.fr

ANDREAS E. KYPRIANOU

Department of Mathematical Sciences, University of Bath, Bath BA2 7AY, UK.

email: a.kyprianou@bath.ac.uk

Submitted April 2, 2007, accepted in final form April 1, 2008

AMS 2000 Subject classification: 60G44, 60H05, 60J55, 60J65

Keywords: Stochastic calculus, local time-space, Itô formula, parabolic generator.

Abstract

The purpose of this note is twofold. Firstly to complete a recent accumulation of results concerning extended version of Itô's formula for any one dimensional Lévy processes, $X$. Secondly, we use the latter to characterise the parabolic generator of $X$

$$
\mathbf{A}:=\left\{(f, g): f(X ., \cdot)-\int_{0}^{\cdot} g\left(X_{s}, s\right) d s \text { is a local martingale }\right\} .
$$

We also establish a necessary condition for a pair of functions to be in the domain of the parabolic generator when $X$ has a Gaussian component.

\section{Parabolic generator of a Lévy process}

Let $\left(X_{t}, t \geq 0\right)$ be a one-dimensional Lévy process defined on the probability space $(\Omega, \mathcal{F}, \mathbb{P})$ satisfying the usual conditions. It admits the following decomposition, the so called Lévy-Itô decomposition, (see for example Bertoin [3] p. 14);

$$
X_{t}=\mu t+\sigma B_{t}+X_{t}^{(2)}+X_{t}^{(3)}
$$

where $\sigma$ and $\mu$ are real numbers, $B$ is a linear Brownian motion, $X_{t}^{(2)}=\sum_{0 \leq s \leq t} \Delta X_{s} 1_{\left(\left|\Delta X_{s}\right| \geq 1\right)}$ and the process $X^{(3)}$ is obtained as the uniform $L^{2}(\mathbb{P})$-limit on compact intervals of time of the sequence $\left(X^{(\epsilon, 3)}, \epsilon>0\right)$ as $\epsilon$ tends to 0 , with

$$
X_{t}^{(\epsilon, 3)}=\sum_{0 \leq s \leq t} \Delta X_{s} 1_{\left(\epsilon<\left|\Delta X_{s}\right| \leq 1\right)}-t \int_{\mathbf{R}} x 1_{\{\epsilon<|x|<1\}} \Pi(d x)
$$


where $\Pi$ is the Lévy measure.

Let us define the parabolic generator, $\mathbf{A}$, of this process to consist of all pairs $(f, g)$ of functions for which

$$
f\left(X_{t}, t\right)-\int_{0}^{t} g\left(X_{s}, s\right) d s
$$

is a local martingale. (Some authors refer to this definition as the extended parabolic generator). The first of our two main objectives in this paper is Theorem 2 below for which the following definition is needed.

Definition 1. Let $\mathbf{F}$ be the set of functions defined on $\mathbf{R} \times \mathbf{R}^{+}$. Then

$\mathcal{D}_{1,1}$ consists of functions $F \in \mathbf{F}$ such that $\partial F / \partial x$ and $\partial F / \partial t$ exist as Radon-Nikodym derivatives with respect to the Lebesgue measure and are locally bounded,

$\mathcal{D}_{2,1}$ consists of functions $F \in \mathbf{F}$ such that $\partial^{2} F / \partial x^{2}$ and $\partial F / \partial t$ exist as Radon-Nikodym derivatives with respect to the Lebesgue measure and are locally bounded,

$\mathcal{I}_{1}$ consists of functions $F \in \mathbf{F}$ such that

$$
\int_{\mathbb{R}} \Pi(d y)|F(x+y, t)-F(x, t)|
$$

is well defined and locally bounded in $(x, t)$ and

$\mathcal{I}_{2}$ consists of functions $F \in \mathbf{F}$ such that $\partial F / \partial x$ exists as a Radon-Nikodym derivative with respect to the Lebesgue measure and

$$
\int_{\mathbb{R}} \Pi(d y)\left|F(x+y, t)-F(x, t)-\frac{\partial F}{\partial x}(x, t) y \mathbf{1}_{(|y|<1)}\right|
$$

is well defined and locally bounded in $(x, t)$.

Let $\mathcal{L}$ be the parabolic integro-differential operator associated with the generator of $X$ so that

$$
\begin{aligned}
\mathcal{L} F(x, t)= & \frac{\partial F}{\partial t}(x, t)+\mu \frac{\partial F}{\partial x}(x, t)+\frac{1}{2} \sigma^{2} \frac{\partial^{2} F}{\partial x^{2}}(x, t) \\
& +\int_{\mathbb{R}}\left\{F(x+y, t)-F(x, t)-y \frac{\partial F}{\partial x}(x, t) \mathbf{1}_{(|y|<1)}\right\} \Pi(d y),
\end{aligned}
$$

whenever $\frac{\partial F}{\partial t}, \frac{\partial F}{\partial x}$ and $\frac{\partial^{2} F}{\partial x^{2}}$ exist as Radon-Nikodym derivatives and the integral is well defined.

Theorem 2. Set $\alpha=1_{\{\sigma \neq 0\}}$ and $\beta=1_{\left\{\sigma=0 ; \int_{(-1,1)}|x| \Pi(d x)=\infty\right\}}$. We have then:

(i) If $F \in \mathcal{D}_{1+\alpha, 1} \cap \mathcal{I}_{1+\beta}$, then (1) is well defined and $(F, \mathcal{L} F) \in \mathbf{A}$

(ii) Assume that $\sigma \neq 0$. Let $F$ be an element of $\mathcal{D}_{1,1} \cap \mathcal{I}_{2}$ such that $\frac{\partial F}{\partial x}$ is continuous. Assume that there exists a continuous function $G$ such that $(F, G)$ belongs to $\mathbf{A}$, then $F$ is in $\mathcal{D}_{2,1} \cap \mathcal{I}_{2}$ and $G=\mathcal{L} F$.

To some extent part (i) is predictable from mathematical 'folklore' for generators but we have been unable to find a similar concise statement in the literature. A natural way to prove part (i) would be to use the infinitesimal generator of the two-dimensional Lévy process $\left(\left(X_{t}, t\right): t \geq 0\right)$ and show using mollification arguments that its domain contains $\mathcal{D}_{1+\alpha, 1} \cap \mathcal{I}_{1+\beta}$. Instead, we 
prefer to obtain Theorem 2 (i) as an immediate consequence of an extended version of the Itô formula (which is introduced in Section 2 and proved in Section 4). Technically, the two methods are equivalent, but the extended Itô formula provides also Theorem 2 (ii) which is a necessary condition on elements of $\mathbf{A}$, and as such, usually quite difficult to obtain. To our knowledge, part (ii) is only known in the literature for the case of Brownian motion; see Wang $[17]$.

\section{Extended Itô formula}

The following theorem completes a recent accumulation of results concerning extended version of Itô's formula for one dimensional Lévy processes. As it has been done in [6], the Itô formula below can easily be extended to multidimensional Lévy processes.

Theorem 3. Suppose that $F \in \mathcal{D}_{1,1}$ and moreover assume that

$$
\int_{|y|<1} \Pi(d y)\left|F(x+y, s)-F(x, s)-\frac{\partial F}{\partial x}(x, s) y\right|
$$

is well defined and locally bounded in $(x, s)$. Then for $t \geq 0$ we have $\mathbb{P}$-almost surely

$$
\begin{aligned}
F\left(X_{t}, t\right)= & F\left(X_{0}, 0\right)+\int_{0}^{t} \frac{\partial F}{\partial t}\left(X_{s}, s\right) d s+\int_{0}^{t} \frac{\partial F}{\partial x}\left(X_{s-}, s\right) d X_{s}-\frac{1}{2} \int_{0}^{t} \int_{\mathbf{R}} \frac{\partial F}{\partial x}(x, s) d L_{s}^{x} \\
& +\sum_{0<s \leq t}\left\{F\left(X_{s}, s\right)-F\left(X_{s-}, s\right)-\frac{\partial F}{\partial x}\left(X_{s-}, s\right) \Delta X_{s}\right\}
\end{aligned}
$$

where $\left(L_{t}^{x}, x \in \mathbb{R}, t \geq 0\right)$ is the semi-martingale local time of $X$.

Note that the third, local time-space integral needs to be understood in an appropriate sense and this is defined in the next section. It is worth remarking at this point in time however that the semi-martingale local time of $X$ is non-zero if and only if a Gaussian component is present $(\sigma \neq 0)$.

The original interest of extended Itô formulae similar to the one above but for the case of Brownian motion was to unify various existing formulae such as the Bouleau-Yor formula [4], the Föllmer-Protter-Shiryaev's formula [12] and the Azéma-Jeulin-Knight-Yor's formula [2]. Naturally from these studies grew a desire to see a generic formula covering all cases; see for example Ghomrasni and Peskir [13], Errami et al. [9]. At the same time, the need for extended versions of the classical Itô formula appeared in the field of optimal stopping problems driven by continuous semi-martingales and free boundary problems. In such cases one typically works with space-time functions which are $C^{2,1}\left(\mathbf{R} \times \mathbf{R}^{+}\right)$except on a set $\left\{(x, t) \in \mathbf{R} \times \mathbf{R}^{+}: x=\right.$ $b(t)\}$ where $b: \mathbf{R}^{+} \mapsto \mathbf{R}$ is a continuous function. Peskir [15, 16] provides the generic form of such formulae for general semi-martingales, also in higher dimensional Euclidian space. Simultaneously developments have appeared in the theory of caustics which have brought with them similar extended versions of Itô's formula; see Elworthy et al. [8]. We mention that Feng and Zhao have constructed integration with respect to local time using Young integrals [10] and rough path integrals [11].

Theorem 3 is devoted to the case of a general one dimensional Lévy processes and extends the recent result of Eisenbaum [6] which covered Lévy processes whose jump component is of bounded variation. In relaxing the assumptions on the jump component, it has become necessary to introduce the condition (2). 


\section{Local time-space calculus}

Let us begin with some notational conventions.

Definition 4. The covariation of two stochastic processes $Y$ and $Z$ on the time interval $[0, t]$ is defined as the following limit, when it exists, in probability

$$
[Y, Z]_{t}=\lim _{n \rightarrow \infty} \sum_{i=1}^{n}\left(Y_{t_{i+1}}-Y_{t_{i}}\right)\left(Z_{t_{i+1}}-Z_{t_{i}}\right)
$$

where the limit is taken over all the sequences of the subdivisions $0=t_{1}<t_{2}<\ldots<t_{n}=t$ such that $\sup _{1 \leq i \leq n}\left|t_{i+1}-t_{i}\right|$ tends to 0 when $n$ tends to $\infty$.

Now let $\left(X_{s}, s \geq 0\right)$ be a general one-dimensional Lévy process. Its semi-martingale local time process $\left(L_{t}^{a}, t \geq 0, a \in \mathbf{R}\right)$ is given by Tanaka's formula. Let $f_{\Delta}$ be a simple function ie there exist a finite sequence $\left(x_{i}\right)_{1 \leq i \leq n}$ of real numbers, a subdivision of $[0,1] \quad\left(s_{j}\right)_{1 \leq j \leq m}$ and a family of real numbers $\left\{f_{i j}, 1 \leq i \leq n, 1 \leq j \leq m\right\}$ such that

$$
f_{\Delta}(x, s)=\sum_{\left(x_{i}, s_{j}\right) \in \Delta} f_{i j} 1_{\left(x_{i}, x_{i+1}\right]}(x) 1_{\left(s_{j}, s_{j+1}\right]}(s)
$$

where $\Delta=\left\{\left(x_{i}, s_{j}\right), 1 \leq i \leq n, 1 \leq j \leq m\right\}$. For such a function integration with respect to $L$, the local time process of $X$, is defined by

$$
\int_{0}^{1} \int_{\mathbf{R}} f_{\Delta}(x, s) d L_{s}^{x}=\sum_{\left(x_{i}, s_{j}\right) \in \Delta} f_{i j}\left(L_{s_{j+1}}^{x_{i+1}}-L_{s_{j}}^{x_{i+1}}-L_{s_{j+1}}^{x_{i}}+L_{s_{j}}^{x_{i}}\right)
$$

A natural problem is to find the set of functions to which this integration could be extended. We do this in the following theorem. To do so we recall a well-known result already used in [6]. The reversed process $\hat{B}=\left(\hat{B}_{t}:=B_{(1-t)}: t \in[0,1]\right)$ is a semi-martingale with respect to to the natural filtration of $\hat{X}=\left(\hat{X}_{t}:=X_{(1-t)-}: t \in[0,1]\right)$. (Recall that $B$ is a standard Brownian motion appearing in the Lévy-Itô decomposition of $X)$. It admits the following decomposition

$$
\hat{B}_{t}=B_{1}+W_{t}-\int_{0}^{t} \frac{\hat{B}_{s}}{1-s} d s
$$

where $\left(W_{s}, 0 \leq s \leq 1\right)$ is a Brownian motion starting from 0 , with respect to the filtration of $\hat{B}$.

Theorem 5. Let $X$ be a linear Lévy process with a Gaussian component equal to $\left(\sigma B_{t}: t \geq 0\right)$ where $\left(B_{t}: t \geq 0\right)$ is a standard Brownian motion and $\sigma$ a real number. Let $f$ be a locally bounded measurable function from $\mathbf{R} \times[0, \infty)$ to $\mathbf{R}$. Then $\int_{0}^{t} \int_{\mathbf{R}} f(x, s) d L_{s}^{x}$ is well-defined and we have :

$$
\int_{0}^{t} \int_{\mathbf{R}} f(x, s) d L_{s}^{x}=\sigma \int_{0}^{t} f\left(X_{s-}, s\right) d B_{s}+\sigma \int_{1-t}^{1} f\left(\hat{X}_{s-}, 1-s\right) d \hat{B}_{s} ; 0 \leq t \leq 1,
$$

moreover

$$
\mathbb{E}\left(\left|\int_{0}^{1} \int_{\mathbf{R}} f(x, s) d L_{s}^{x}\right|\right) \leq|\sigma|\|f\|
$$

where for any measurable function $f: \mathbf{R} \times[0,1] \mapsto \mathbf{R}$ the norm $\|\cdot\|$ is defined by

$$
\|f\|=2 E\left(\int_{0}^{1} f^{2}\left(X_{s}, s\right) d s\right)^{1 / 2}+\mathbb{E}\left(\int_{0}^{1}\left|f\left(X_{s}, s\right) \frac{B_{s}}{s}\right| d s\right) .
$$


Proof: The reversed Lévy process $\hat{X}$ is a semi-martingale and as such admits a local time process on the time interval $[0,1]$ that we denote $\left(\hat{L}_{t}^{x}, x \in \mathbf{R}\right)$. Thanks to the occupation time formula one has

$$
L_{t}^{x}=\hat{L}_{1}^{x}-\hat{L}_{1-t}^{x} .
$$

Thanks to Tanaka's formula and (7) we have

$$
\begin{aligned}
-L_{t}^{a}= & \int_{0}^{t} 1_{\left(X_{s}->a\right)} d X_{s}+\int_{1-t}^{1} 1_{\left(\hat{X}_{s}->a\right)} d \hat{X}_{s} \\
& +\sum_{0 \leq s \leq t}\left\{1_{\left(X_{s}>a\right)}-1_{\left(X_{s-}>a\right)}\right\} \Delta X_{s}
\end{aligned}
$$

for any $t$ in $(0,1]$. From the Lévy-Itô decomposition recalled in Section 1, (8) becomes

$$
\begin{aligned}
-L_{t}^{a}= & \sigma \int_{0}^{t} 1_{\left(X_{s}->a\right)} d B_{s}+\sigma \int_{1-t}^{1} 1_{\left(\hat{X}_{s}->a\right)} d \hat{B}_{s}+\int_{0}^{t} 1_{\left(X_{s}->a\right)} d X_{s}^{(3)} \\
& +\int_{1-t}^{1} 1_{\left(\hat{X}_{s}->a\right)} d \hat{X}_{s}^{(3)}+\sum_{0 \leq s \leq t}\left\{1_{\left(X_{s}>a\right)}-1_{\left(X_{s-}>a\right)}\right\} \Delta X_{s} 1_{\left(\left|\Delta X_{s}\right|<1\right)} .
\end{aligned}
$$

Next we develop the term $\int_{0}^{t} 1_{\left(X_{s}->a\right)} d X_{s}^{(3)}+\int_{1-t}^{1} 1_{\left(\hat{X}_{s->a)}\right.} d \hat{X}_{s}^{(3)}$. On account of the definition of $X^{(3)}$, it is equal to the limit in $L^{2}(\mathbb{P})$ of

$$
\begin{aligned}
& \sum_{0 \leq s \leq t} 1_{\left(X_{s}->a\right)} \Delta X_{s} 1_{\left(\epsilon<\left|\Delta X_{s}\right| \leq 1\right)}-\int_{\mathbf{R}} x 1_{\{\epsilon<|x|<1\}} \Pi(d x) \int_{0}^{t} 1_{\left(X_{s}->a\right)} d s \\
& -\sum_{0 \leq s \leq t} 1_{\left(X_{s}>a\right)} \Delta X_{s} 1_{\left(\epsilon<\left|\Delta X_{s}\right| \leq 1\right)}+\int_{\mathbf{R}} x 1_{\{\epsilon<|x|<1\}} \Pi(d x) \int_{0}^{t} 1_{\left(X_{s}>a\right)} d s .
\end{aligned}
$$

which is equal to the limit of $\sum_{0 \leq s \leq t}\left\{1_{\left(X_{s-}>a\right)}-1_{\left(X_{s}>a\right)}\right\} \Delta X_{s} 1_{\left(\epsilon<\left|\Delta X_{s}\right|<1\right)}$. Consequently, we finally obtain

$$
-L_{t}^{a}=\sigma \int_{0}^{t} 1_{\left(X_{s}->a\right)} d B_{s}+\sigma \int_{1-t}^{1} 1_{\left(\hat{X}_{s}->a\right)} d \hat{B}_{s}
$$

and similarly

$$
L_{t}^{a}=\sigma \int_{0}^{t} 1_{\left(X_{s}-\leq a\right)} d B_{s}+\sigma \int_{1-t}^{1} 1_{\left(\hat{X}_{s}-\leq a\right)} d \hat{B}_{s} .
$$

Let $f_{\Delta}$ be a simple function. Thanks to (10) and (4) we obtain

$$
\mathbb{E}\left(\left|\int_{0}^{1} \int_{\mathbf{R}} f_{\Delta}(x, s) d L_{s}^{x}\right|\right) \leq|\sigma|\left\|f_{\Delta}\right\|
$$

which allows to obtain a consistent definition for $\int_{0}^{t} \int_{\mathbf{R}} f(x, s) d L_{s}^{x}, t \geq 0$, for $f$ such that $\|f\|<$ $\infty$, which satisfies (6). One may then enlarge then this stochastic integration to measurable locally bounded functions as was done in [6].

Definition 6. The local time of $X$ along any Borelian curve $(b(t), 0 \leq t \leq 1)$ is defined by

$$
L_{t}^{b(\cdot)}=\int_{0}^{t} \int_{\mathbf{R}} 1_{(-\infty, b(s))}(x) d L_{s}^{x}, t \geq 0 .
$$


Naturally, unless $\sigma \neq 0$ the above integral is identically zero as a process in time.

The next lemma gives some rules for computing integrals with respect to local times. Its proof is similar to the proofs of Lemma 2.3 and Lemma 4.1 in [7] and hence is omitted.

Lemma 7. Suppose that $\sigma \neq 0$.

(i) Let $f$ be a continuous function on $\mathbf{R} \times[0,1]$ then for $0 \leq t \leq 1$

$$
\int_{0}^{t} \int_{\mathbf{R}} f(x, s) d L_{s}^{x}=-[f(X ., .), B .]_{t} .
$$

(ii) Let $f$ be a function on $\mathbf{R} \times[0,1]$ admitting a locally bounded Radon-Nikodym derivative $\partial f / \partial x$ then for $0 \leq t \leq 1$

$$
\int_{0}^{t} \int_{\mathbf{R}} f(x, s) d L_{s}^{x}=-\int_{0}^{t} \frac{\partial f}{\partial x}\left(X_{s}, s\right) d[X]_{s}^{c} .
$$

(iii) Let b be a continuous function from $\mathbf{R}_{+}$to $\mathbf{R}$. Let $f$ be a continuous function on $\mathbf{R} \times[0,1]$, admitting a continuous derivative $\partial f / \partial x$. Then we have for $0 \leq t \leq 1$,

$$
\begin{aligned}
\int_{0}^{t} \int_{\mathbf{R}} f(x, s) 1_{(x \leq b(s))} d L_{s}^{x}= & \int_{0}^{t} f(b(s), s) d_{s} L_{s}^{b(.)} \\
& -\int_{0}^{t} \frac{\partial f}{\partial x}\left(X_{s}, s\right) 1_{\left(X_{s} \leq b(s)\right)} d[X]_{s}^{c} .
\end{aligned}
$$

The following corollary presents solutions to the problem

$$
\int_{0}^{t} \int_{\mathbf{R}} f(x, s) d L_{s}^{x}=0, \quad 0 \leq t \leq 1 .
$$

Corollary 8. Suppose that $\sigma \neq 0$.

(i) If $f$ is a solution of (11) admitting a continuous derivative with respect to $x$ then the function $\frac{\partial f}{\partial x}$ is identically equal to 0 .

(ii) If $f(x, s)=F(s)$, then $\int_{0}^{t} \int_{\mathbf{R}} f(x, s) d L_{s}^{x}=0$

(iii) Let $f$ be a continuous solution of (11) then $\frac{\partial f}{\partial x}$ exists and is identically equal to 0 .

Proof of Corollary 8: (i) We have $\int_{0}^{t} \frac{\partial f}{\partial x}\left(X_{s}, s\right) d s=0$ for every $t$ in [0,1]. Hence since $X$ is right continuous $\frac{\partial f}{\partial x}\left(X_{s}, s\right)=0$ for every $s \in[0,1]$. This implies that $\mathbb{E}\left(\left|\frac{\partial f}{\partial x}\left(X_{s}, s\right)\right|\right)=0$. Since $X_{s}$ has a density with respect to the Lebesgue measure, we conclude that $\frac{\partial f}{\partial x}(x, s)=0$ $d x$ a.e.. But $\frac{\partial f}{\partial x}$ is continuous hence $\frac{\partial f}{\partial x}$ is identically equal to 0 .

(ii) In this case $f$ obviously admits a partial derivative with respect to $x$ which is identically equal to 0 . We obtain the conclusion thanks to Lemma 7 (ii).

(iii) Thanks to Theorem 7 , the equality (11) is equivalent to

$$
\int_{0}^{t} f\left(X_{s-}, s\right) d B_{s}+\int_{1-t}^{1} f\left(\hat{X}_{s-}, 1-s\right) d \hat{B}_{s}=0 ; 0 \leq t \leq 1 .
$$


We assume first that $f$, solution of (11) is bounded. Since the process $\left(M_{t}, 0 \leq t \leq 1\right)=$ $\left(\int_{0}^{t} f\left(X_{s-}, s\right) d B_{s}, 0 \leq t \leq 1\right)$ is a martingale with respect to $\mathcal{F}_{t}=\sigma\left(X_{u} ; 0 \leq u \leq t\right)$, then this also true for the process $\left(\int_{1-t}^{1} f\left(\hat{X}_{s-}, 1-s\right) d \hat{B}_{s}, 0 \leq t \leq 1\right)$. We set $K_{t}=\int_{0}^{t} f\left(\hat{X}_{s-}, 1-\right.$ $s) d \hat{B}_{s}, 0 \leq t \leq 1$. Note that $\left(K_{t}, 0 \leq t \leq 1\right)$ is a semi-martingale with respect to $\hat{\mathcal{F}}_{t}=$ $\sigma\left(\hat{X}_{u} ; 0 \leq u \leq t\right)$. Thanks to (12), we have for every $C^{2}$ - function $h$ with compact support

$$
\int_{0}^{t} h\left(X_{s-}\right) d M_{s}-\int_{0}^{t} h\left(X_{s-}\right) d K_{1-s}=0, \quad 0 \leq t \leq 1 .
$$

By using an almost classical argument by now we have

$$
\int_{0}^{t} h\left(X_{s-}\right) d K_{1-s}=[h(\hat{X}), K]_{1-t}-[h(\hat{X}), K]_{1}-\int_{1-t}^{1} h\left(\hat{X}_{s-}\right) d K_{s} .
$$

Expanding $h\left(\hat{X}_{t}\right)$ thanks to the classical Itô formula, we can hence rewrite (13) as follows

$$
\begin{gathered}
\int_{0}^{t} h\left(X_{s-}\right) f\left(X_{s-}, s\right) d B_{s}+\int_{1-t}^{1} h\left(\hat{X}_{s-}\right) f\left(\hat{X}_{s-}, s\right) d W_{s} \\
-\int_{1-t}^{1} h\left(\hat{X}_{s-}\right) f\left(\hat{X}_{s-}, s\right) \frac{\hat{B}_{s}}{1-s} d s+\int_{1-t}^{1} h^{\prime}\left(\hat{X}_{s-}\right) f\left(\hat{X}_{s-}, s\right) d s=0
\end{gathered}
$$

Taking the expectation on both sides of the above equation, we obtain:

$$
\int_{0}^{t} d s \mathbb{E}\left[f\left(X_{s}, s\right)\left\{h^{\prime}\left(X_{s}\right)-\frac{B_{s}}{s} h\left(X_{s}\right)\right\}\right]=0,0 \leq t \leq 1
$$

Denoting by $\left.\left(p_{s}(x), x \in \mathbf{R}\right)\right)$ the density of $X_{s}$ with respect to the Lebesgue measure (which exists as $\sigma \neq 0)$ and setting: $g(x, s)=\mathbb{E}\left(B_{s} \mid X_{s}=x\right)$, we obtain

$$
\int_{0}^{t} d s \int_{\mathbf{R}} d x p_{s}(x) f(x, s)\left\{h^{\prime}(x)-\frac{g(x, s)}{s} h(x)\right\}=0,0 \leq t \leq 1
$$

By a simple integration by part, we have

$$
\int_{\mathbf{R}} d x p_{s}(x) f(x, s) \frac{g(x, s)}{s} h(x)=-\int_{\mathbf{R}} d x h^{\prime}(x) v_{s}(x)
$$

where $v_{s}(x)=\int_{-\infty}^{x} p_{s}(y) f(y, s) \frac{g(y, s)}{s} d y$ (note that $v_{s}(x)$ is a continuous function of $(x, s)$ ). Thanks to (14), this implies that

$$
\int_{0}^{t} d s \int_{\mathbf{R}} d x h^{\prime}(x)\left(v_{s}(x)+p_{s}(x) f(x, s)\right)=0,0 \leq t \leq 1
$$

and since this is true for every continuous function with compact support, we obtain by Fubini

$$
\int_{0}^{t} d s\left(v_{s}(x)+p_{s}(x) f(x, s)\right)=0, d x \text { a.e. }
$$

As $\epsilon$ tends to 0 one obtains $d x$ a.e.

$$
\frac{1}{\epsilon} \int_{t}^{t+\epsilon} d s\left(v_{s}(x)+p_{s}(x) f(x, s)\right) \rightarrow v_{t}(x)+p_{t}(x) f(x, t)
$$


and consequently $p_{t}(x) f(x, t)=-v_{t}(x) d x$ a.e. $d t$ a.e.. By continuity this last identity is true for every $(x, t) \in \mathbf{R} \times[0,1]$. We remark that the function $p_{t}(x)$ admits a derivative with respect to $x$ ( actually $p_{t}(x)=\mathbb{E}\left(\frac{1}{\sqrt{2 \pi \sigma^{2} t}} \exp \left\{-\frac{\left(x-Y_{t}\right)^{2}}{2 \sigma^{2} t}\right\}\right)$ where $\left.Y_{t}=X_{t}-\sigma B_{t}\right)$. This implies that $f$ admits a continuous derivative with respect to $x$. Thanks to (i) we conclude that this derivative is equal to 0 .

\section{Proof of Theorem 3}

The statement of Theorem 3 for the case that $X$ is a compound Poisson process with drift is a trivial consequence of the use of a telescopic sum over jump times (cf. Kyprianou and Surya [14] ) and hence is excluded from the proof. For the case $X$ has no Brownian component $(\sigma=0)$ and hence its characteristics are $(\mu, 0, \Pi(d x))$, we call $X^{\epsilon}$ the Lévy process with characteristics $\left(\mu, 0,1_{\{|x| \geq \epsilon\}} \Pi(d x)\right)$, One can take advantage of the previous remark and notice that for $F$ satisfying (2) formula (3) is available for $X^{\epsilon}$ for every $\epsilon>0$. By letting then $\epsilon$ tend to 0 one easily obtains (3) for $X$ since the convergence concerns only the domain of integration $\{|x| \geq \epsilon\}$.

From now on, we assume that $\sigma \neq 0$. We first assume that the partial derivatives are bounded. For $n \in \mathbf{N}^{*}$, let $F_{n}$ be the function defined by

$$
F_{n}(x, t)=\iint_{\mathbf{R}^{2}} F(x-(y / n), t-(s / n)) f(y) h(s) d y d s
$$

where $f$ and $h$ are two $\mathcal{C}^{\infty}$ functions from $\mathbf{R}$ to $\mathbf{R}_{+}$with compact support, such that $\int_{\mathbf{R}} f(y) d y=$ $1=\int_{\mathbf{R}} h(y) d y$.

The usual Itô formula applies to $F_{n}\left(X_{t}, t\right)$. Letting $n$ tend to $\infty$, one obtains using the same arguments developed in [5] and [6] the convergence of each term of the expansion of $F_{n}\left(X_{t}, t\right)$ to the corresponding expression with $F$ instead of $F_{n}$, except for two terms that require an adapted treatment

$$
\int_{0}^{t} \frac{\partial F_{n}}{\partial x}\left(X_{s-}, s\right) d X_{s}^{(3)}
$$

and

$$
\Sigma(n)=\sum_{0<s \leq t}\left\{F_{n}\left(X_{s}, s\right)-F_{n}\left(X_{s-}, s\right)-\frac{\partial F_{n}}{\partial x}\left(X_{s-}, s\right) \Delta X_{s}\right\} 1_{\left(\left|\Delta X_{s}\right| \leq 1\right)}
$$

To this end, let $N(d s, d y)$ be the Poisson random measure associated with jumps of $X$ and define the compensated Poisson measure : $\tilde{N}(d s, d y)=N(d s, d y)-d s \Pi(d y)$. Let $\mathcal{H}_{2}$ be the space of all equivalence classes of mappings $G$ from $\mathbf{R} \times[0,1]$ to $\mathbf{R}$ which coincide a.e. with respect to $d s \Pi(d y) \times \mathbb{P}$ such that $G$ is predictable and

$$
\int_{0}^{1} \int_{\mathbf{R}} \mathbb{E}\left[(G(x, t))^{2}\right] d t \Pi(d y)<\infty .
$$

We define a norm on $\mathcal{H}_{2}$ by setting

$$
\|G\|_{\mathcal{H}_{2}}=\left[\int_{0}^{1} \int_{\mathbf{R}} \mathbb{E}\left[(G(x, t))^{2}\right] d t \Pi(d y)\right]^{1 / 2} .
$$

Since

$$
\mathbb{E}\left[\left(\int_{0}^{1} \int_{\mathbf{R}} G(x, t) \tilde{N}(d s, d y)\right)^{2}\right]=\|G\|_{\mathcal{H}_{2}}^{2}
$$


the mapping

$$
G \mapsto \int_{0}^{1} \int_{\mathbf{R}} G(x, t) \tilde{N}(d s, d y)
$$

is an isometry from $\mathcal{H}_{2}$ into $L^{2}(\mathbb{P})$ (see for example Applebaum p. 199 [1]).

Note that

$$
\int_{0}^{t} \frac{\partial F_{n}}{\partial t}\left(X_{s-}, s\right) d X_{s}^{(3)}=\int_{0}^{t} \int_{\mathbf{R}} \frac{\partial F_{n}}{\partial t}\left(X_{s-}, s\right) y 1_{(|y|<1)} \tilde{N}(d s, d y) .
$$

Since the function $1_{(|y|<1)} y^{2}$ is $\Pi(d y)$-integrable, we obtain by dominated convergence

$$
\int_{0}^{t} \frac{\partial F_{n}}{\partial t}\left(X_{s-}, s\right) d X_{s}^{(3)} \underset{n \rightarrow \infty}{\stackrel{L^{2}(\mathbb{P})}{\longrightarrow}} \int_{0}^{t} \frac{\partial F}{\partial t}\left(X_{s-}, s\right) d X_{s}^{(3)}
$$

Hence, all the terms in the Itô's formula for $F_{n}\left(X_{t}, t\right)$, with the exception of $\Sigma(n)$, converge as $n$ tends to $\infty$. Hence $\Sigma(n)$ converges too in $L^{1}(\mathbb{P})$ to a limit that we denote by $\Sigma$.

The problem now is to find an appropriate expression for $\Sigma$. We set

$$
H(y, s)=\left\{F\left(X_{s-}+y, s\right)-F\left(X_{s-}, s\right)-\frac{\partial F}{\partial x}\left(X_{s-}, s\right) y\right\} 1_{(|y|<1)}
$$

and

$$
H_{n}(y, s)=\left\{F_{n}\left(X_{s-}+y, s\right)-F_{n}\left(X_{s-}, s\right)-\frac{\partial F_{n}}{\partial x}\left(X_{s-}, s\right) y\right\} 1_{(|y|<1)} .
$$

Note that

$$
|H(y, s)| \leq 2 \sup _{x \in \mathbf{R}}\left|\frac{\partial F}{\partial x}(x, s)\right| 1_{(|y|<1)}|y|
$$

Since the function $1_{(|y|<1)} y^{2}$ is $\Pi(d y)$-integrable, the function $H$ is thus an element of $\mathcal{H}_{2}$. The same remark is available for the function $H_{n}$. Moreover by dominated convergence, we have $H_{n} \rightarrow H$ in the $\|\cdot\|_{\mathcal{H}_{2}}$ norm as $n \rightarrow \infty$ and consequently

$$
\int_{0}^{1} \int_{\mathbf{R}} H_{n}(x, t) \tilde{N}(d s, d y) \underset{n \rightarrow \infty}{\stackrel{L^{2}(\mathbb{P})}{\longrightarrow}} \int_{0}^{1} \int_{\mathbf{R}} H(x, t) \tilde{N}(d s, d y) .
$$

Note also that that $\Sigma(n)=\int_{0}^{t} \int_{\mathbf{R}} H_{n}(y, s) N(d s, d y)$, hence

$$
\Sigma(n)=\int_{0}^{t} \int_{\mathbf{R}} H_{n}(y, s) \tilde{N}(d s, d y)+\int_{0}^{t} \int_{\mathbf{R}} H_{n}(y, s) d s \Pi(d y) .
$$

The convergence of $\Sigma(n)$ thus boils down to the convergence of $\int_{0}^{t} \int_{\mathbf{R}} H_{n}(y, s) d s \Pi(d y)$ as $n$ tends to $\infty$. We have

$$
\begin{aligned}
\int_{0}^{t} \int_{\mathbf{R}} H_{n}(y, s) d s \Pi(d y)= & \int_{0}^{t} \int_{\{|y|<1\}} \int_{\mathbf{R}} \int_{\mathbf{R}} f(z) h(u)\left\{F\left(X_{s-}+y-\frac{z}{n}, s-\frac{u}{n}\right)\right. \\
& \left.-F\left(X_{s-}-\frac{z}{n}, s-\frac{u}{n}\right)-\frac{\partial F}{\partial x}\left(X_{s-}-\frac{z}{n}, s-\frac{u}{n}\right) y\right\} d z d u d s \Pi(d y) \\
= & \int_{\mathbf{R}} \int_{\mathbf{R}} f(z) h(u) d z d u\left[\int _ { 0 } ^ { t } \int _ { \{ | y | < 1 \} } \left\{F\left(X_{s-}+y-\frac{z}{n}, s-\frac{u}{n}\right)\right.\right. \\
& \left.\left.-F\left(X_{s-}-\frac{z}{n}, s-\frac{u}{n}\right)-\frac{\partial F}{\partial x}\left(X_{s-}-\frac{z}{n}, s-\frac{u}{n}\right) y\right\} d s \Pi(d y)\right]
\end{aligned}
$$


where the second equality follows by a simple application of Fubini's Theorem, allowed by the fact that

$$
\int_{|y|<1} \Pi(d y)\left|F\left(X_{s-}+y-\frac{z}{n}, s-\frac{u}{n}\right)-F\left(X_{s-}-\frac{z}{n}, s-\frac{u}{n}\right)-\frac{\partial F}{\partial x}\left(X_{s-}-\frac{z}{n}, s-\frac{u}{n}\right) y\right|
$$

is uniformly bounded in $(z, u, s)$ inside $\operatorname{supp}(f) \times \operatorname{supp}(h) \times[0,1]$. Hence

$$
\int_{0}^{t} \int_{\mathbf{R}} H_{n}(y, s) d s \Pi(d y) \underset{n \rightarrow \infty}{\stackrel{L^{1}(\mathbb{P})}{\longrightarrow}} \int_{0}^{t} \int_{\{|y|<1\}}\left\{F\left(X_{s-}+y, s\right)-F\left(X_{s-}, s\right)-\frac{\partial F}{\partial x}\left(X_{s-}, s\right) y\right\} d s \Pi(d y) .
$$

We obtain from (17) and (18)

$\Sigma=\int_{0}^{t} \int_{\mathbf{R}} H(y, s) \tilde{N}(d s, d y)+\int_{0}^{t} \int_{\{|y|<1\}}\left\{F\left(X_{s-}+y, s\right)-F\left(X_{s-}, s\right)-\frac{\partial F}{\partial x}\left(X_{s-}, s\right) y\right\} d s \Pi(d y)$

and hence $\Sigma=\int_{0}^{t} \int_{\mathbf{R}} H(y, s) N(d s, d y)$, or equivalently

$$
\Sigma=\sum_{0<s \leq t}\left\{F\left(X_{s}, s\right)-F\left(X_{s-}, s\right)-\frac{\partial F}{\partial x}\left(X_{s-}, s\right)\right\} \Delta X_{s} 1_{\left(\left|\Delta X_{s}\right|<1\right)}
$$

which ends the proof of Theorem 3 in the case of bounded derivatives.

The extension to the case when $\partial F / \partial x$ and $\partial F / \partial t$ are only locally bounded functions, easily follows as in [6].

\section{Remarks on the extended Itô formula}

One may remove the integral test (2) in Theorem 3 with a stronger assumption of Lipschitz continuity as follows.

Corollary 9. Let $F$ be a function defined on $\mathbf{R} \times \mathbf{R}^{+}$such that $\partial F / \partial x$ and $\partial F / \partial t$ exist as Radon-Nikodym derivatives with respect to the Lebesgue measure and are locally bounded. Moreover we assume that $\partial F / \partial x(., s)$ is Lipschitz uniformly in $s \in(0,1]$. Then the conclusion of Theorem 3 follows.

The assumptions in the corollary above may be considered as, in principle, quite strong. They suffice however to address the Errami-Russo-Vallois formula discussed below. An example of a function which does not satisfy the Lipschitz condition above but for which (2) is satisfied is also discussed below.

The Errami-Russo-Vallois formula: Theorem 3 extends the Itô formula of Errami et al. (Theorem 3.8 in [9]) to Lipschitz functions. Indeed in [9], it is assumed that there exists $\lambda \in[0,1)$ such that

$$
\int_{\mathbb{R}}|x|^{1+\lambda} \Pi(d x)<\infty
$$

and the function $\partial F / \partial x$ is Hölder continuous with exponent $\lambda$. Assume instead that $\partial F / \partial x$ is Lipschitz, then Theorem 3 and Lemma 2 (i) give the exact formulation of the Itô formula of Errami et al. [9] but for the case $\lambda=1$.

Peskir's formula: Peskir's formula concerns continuous semi-martingales. It has been extended to Lévy processes $X$ such that $\sum_{0 \leq s \leq 1}\left|\Delta X_{s}\right|<\infty$ in [6]. The same arguments and the 
proof of Theorem 3 allow to write this formula for any Lévy process for which $X_{t}$ that has a density with respect to Lebesgue measure for all $t>0$ (for example when $\sigma \neq 0$ ). Specifically let $F$ be a continuous function defined as follows. Let $b$ be a continuous function from $\mathbf{R}_{+}$to R. Set :

$$
\begin{aligned}
& C=\left\{(x, s) \in \mathbf{R} \times \mathbf{R}_{+}: x<b(s)\right\} \\
& D=\left\{(x, s) \in \mathbf{R} \times \mathbf{R}_{+}: x>b(s)\right\}
\end{aligned}
$$

We assume that $F$ is $C^{2,1}$ on $\bar{C}$ and on $\bar{D}$. We set $F_{1}=F_{\left.\right|_{C}}$ and $F_{2}=F_{\left.\right|_{D}}$.

$$
\begin{aligned}
F\left(X_{t}, t\right) & =F\left(X_{0}, 0\right)+\int_{0}^{t} \frac{\partial F}{\partial x}\left(X_{s-}, s\right) d X_{s} \\
& +\int_{0}^{t} \frac{\partial F_{1}}{\partial t}\left(X_{s}, s\right) 1_{\left(X_{s}<b(s)\right)} d s+\int_{0}^{t} \frac{\partial F_{2}}{\partial t}\left(X_{s}, s\right) 1_{\left(X_{s} \geq b(s)\right)} d s \\
& +\frac{1}{2} \int_{0}^{t}\left(\frac{\partial^{2} F_{1}}{\partial x^{2}}\left(X_{s}, s\right) 1_{(x<b(s))}+\frac{\partial^{2} F_{2}}{\partial x^{2}}\left(X_{s}, s\right) 1_{(x \geq b(s))}\right) d[X]_{s}^{c} \\
& +\frac{1}{2} \int_{0}^{t}\left(\frac{\partial F_{2}}{\partial x}-\frac{\partial F_{1}}{\partial x}\right)(b(s), s) d_{s} L_{s}^{b(.)} \\
& +\sum_{0<s \leq t}\left\{F\left(X_{s}, s\right)-F\left(X_{s-}, s\right)-\frac{\partial F}{\partial x}\left(X_{s-}, s\right) \Delta X_{s}\right\}
\end{aligned}
$$

which is precisely Peskir's formula but for the non-continuous semi-martingale $X$. Our proof also relaxes the assumption on $b$. Indeed, while Peskir's formula requires that $b$ is continuous with bounded variations, the proof given above requires only that $b$ is continuous.

\section{Proof of Theorem 2}

(i) is an immediate consequence of Theorem 3 and Lemma 7 (ii).

(ii) We use Theorem 3 and the assumptions on $F$ to claim that there exists a continuous function $h$ on $\mathbf{R} \times[0,1]$ such that:

$$
\int_{0}^{t} \int_{\mathbf{R}} \frac{\partial F}{\partial x}(x, s) d L_{s}^{x}=-\int_{0}^{t} h\left(X_{s}, s\right) d s, 0 \leq t \leq 1 .
$$

We set $H(x, s)=\int_{0}^{x} h(y, s) d y$. Thanks to Lemma 9 (ii), we have:

$$
-\int_{0}^{t} h\left(X_{s}, s\right) d s=\int_{0}^{t} \int_{\mathbf{R}} H(x, s) d L_{s}^{x}
$$

Hence we obtain

$$
\int_{0}^{t} \int_{\mathbf{R}} \frac{\partial F}{\partial x}(x, s) d L_{s}^{x}=\int_{0}^{t} \int_{\mathbf{R}} H(x, s) d L_{s}^{x}
$$

Setting $f=\frac{\partial F}{\partial x}-H$, we have now to solve the problem:

$$
\int_{0}^{t} \int_{\mathbf{R}} f(x, s) d L_{s}^{x}=0,0 \leq t \leq 1
$$

Corollary 8 (iii) implies that the function $\frac{\partial F}{\partial x}-H$ admits a partial derivative with respect to $x$ which is identically equal to 0 and hence $\frac{\partial^{2} F}{\partial x^{2}}$ exists and is equal to $h$.

The authors would like to acknowledge the support of NWO grant number 613.000.310. 


\section{References}

[1] Applebaum D. (2004) : Lévy processes and stochastic calculus. Cambridge University Press. MR2072890

[2] Azéma J., Jeulin T., Knight F., Yor M. (1998) : Quelques calculs de compensateurs impliquant l'injectivé de certains processus croissantes. Séminaire de Probabilités XXXII (Lecture Notes in Mathematics). 1686, 316-327. MR1651223

[3] Bertoin J. (1996) : Lévy processes. Cambridge University Press. MR1406564

[4] Bouleau N., Yor M. (1981) : Sur la variation quadratique des temps locaux de certaines semi-martingales .C.R.Acad. Sc. Paris 292 (491-494). MR0612544

[5] Eisenbaum N. (2000) : Integration with respect to local time. Potential Analysis 13 (303328). MR1804175

[6] Eisenbaum N. (2006) : Local time-space stochastic calculus for Lévy processes.Stoch.Proc. and Appl.,116, 5, 757-778. MR2218334

[7] Eisenbaum N. (2007) : Local time-space calculus for reversible semi-martingales. Séminaire de Probab. XL, Lecture Notes in Maths 1899, 137-146.

[8] Elworthy K.D., Truman A., Zhao H.Z. (2007) : Stochastic elementary formula and asymptotics with caustics. I. One-dimensional linear heat equations. Séminaire de Probab. XL, Lecture Notes in Maths 1899, 117-136.

[9] Errami M., Russo F., Vallois P. (2002) : Itô formula for $C^{1, \lambda}$-functions of a càdlàg process.Probab. Theory Relat. Fields 122 (191-221). MR1894067

[10] Feng C. and Zhao H. (2006) : Two-parameter $p$, $q$-variation paths and integrations of local times. Potential Anal. 25, 2, 165-204 . MR2238942

[11] Feng C. and Zhao H. (2008) : Rough paths integral of local time. To appear in C. R. Acad. Sci. Paris, Ser. I.

[12] Föllmer H., Protter P., Shiryayev A.N. (1995) : Quadratic covariation and an extension of Itô's formula. Bernoulli,1 (1/2) (149-169). MR1354459

[13] Ghomrasni R., Peskir G. (2003) : Local time-space calculus and extensions of Itô's formula. Proc. High Dim. Probab. III (Sandbjerg 2002) Prog.Probab. 55, Birkhäser Basel, 177-192. MR2033888

[14] Kyprianou, A.E., Surya, B.A. (2005) A note on the change of variable formula with local time-space for bounded variation Lévy processes. Séminaire de Probab. XL, Lecture Notes in Maths 1899, 97-104.

[15] Peskir G. (2005) : A change-of-variable formula with local time on curves. J. Theoret. Probab. 18 (499-535) MR2167640

[16] Peskir, G. (2006) : A change-of-variable formula with local time on surfaces. Séminaire de Probab. XL, Lecture Notes in Maths 1899, 69-96.

[17] Wang A. T. (1977) : Quadratic variation of functionals of Brownian motion. Ann. of Probab. 5 (756 - 769). MR0445622 